\title{
Logiques territoriales et aménagement durable du territoire : quelles règles de coordination et de décision?
}

\section{Sustainable land use planning and logic: What rules of coordination and decision making?}

\author{
Éric Plottu ${ }^{1}$ et Béatrice Plottu*2 \\ Économiste \\ ${ }^{I}$ Agence de l'Environnement et de la Maîtrise de l'Energie (ADEME) \\ 20 avenue du Grésillé BP 90406, 49004 Angers cedex 01 \\ ${ }^{2}$ Maître de conférences en économie \\ AGROCAMPUS OUEST - Centre d'Angers, Institut National d'Horticulture et de Paysage \\ GRANEM (Groupe de Recherche ANgevin en Economie et Management),UMR-MA n ${ }^{\circ} 49$ \\ (AGROCAMPUS OUEST- Université d'Angers) \\ 2, rue Le Nôtre 49045 Angers cedex 01
}

\section{Résumé}

L'imbrication spatiale des territoires fait que tout projet d'aménagement d'un territoire est aussi un projet d'aménagement des territoires venant renforcer ou contrarier les objectifs et schémas d'aménagement d'autres échelons territoriaux. La question se pose alors, pour un aménagement « ordonné » du territoire, de la coordination des différentes logiques territoriales, et de la priorité d'action donnée à telle ou telle initiative. Une première règle possible de coordination et de priorité d'action est fournie par le principe de hiérarchie des systèmes décideurs. La caractérisation du territoire comme système hiérarchique multi-échelons ouvre la voie à une seconde règle possible basée sur la hiérarchie des niveaux d'objectifs poursuivis par les différents systèmes territoriaux . Cette

*Adresse email : beatrice.plottu@agrocampus-ouest.fr doi:10.3166/ges.11.283-299 @ 2009 Lavoisier, Paris. Tous droits réservés. 
seconde règle est intéressante notamment dans une optique de développement durable où il peut s'agir de distinguer la nature et l'importance des enjeux liés à telle ou telle option d'aménagement

(c) 2009 Lavoisier, Paris. Tous droits réservés.

\section{Summary}

The geographical interweaving of landspace means that any planning project reinforces or contradicts planning objectives and diagrams on other landspace levels. For this reason, we can raise the question for "ordered" landspace planning, with coordination of different geographical logic and a priority of action to be given to such or such an initiative. A first possible rule for coordination and priority of action is provided by the principle of hierarchy of deciding systems. The characterisation of landspace as a multi-level hierarchical system opens the way for a second possible rule based on the hierarchy of different levels of objectives pursued by different geographical systems. This second rule is interesting, in particular in a sustainable development framework where it can be used to distinguish the nature and importance of the stakes linked to such and such a planning option.

(C) 2009 Lavoisier, Paris. Tous droits réservés.

Mots clés : aménagement durable du territoire, règles de coordination, système hiérarchique multi-échelons, logiques territoriales

Keywords: sustainable land use planning, rules of coordination, multi-level hierarchic system, land use logic.

\section{Introduction}

L'imbrication spatiale des territoires fait que tout projet d'aménagement d'un territoire est aussi un projet d'aménagement des territoires venant renforcer ou contrarier les objectifs et schémas d'aménagement d'autres échelons territoriaux. L'aménagement de l'espace s'inscrit alors dans un cadre décisionnel complexe et s'apparente à un jeu de conflits/coopérations entre systèmes décideurs (Mesarovic, Macko, Takahara, 1980) de différents territoires.

Ces jeux d'acteurs engendrent nombre de conflits autour de projets d'aménagement du territoire et d'environnement. L'analyse des conflits fait l'objet d'une littérature pluridisciplinaire abondante, notamment l'analyse des relations entre conflits et territorialités. Les conflits comme révélateurs d'identités territoriales ou producteurs de nouvelles territorialités (Melé, Larrue, Rosenberg, 2003) sont ainsi au cœur de l'approche géographique. Ces préoccupations rejoignent celles de travaux menés en sociologie politique et science politique qui étudient les conflits sous l'angle de la territorialisation de l'action publique.

Dans une perspective plus amont, d'autres travaux en économie et aménagement de l'espace (Billé et Mermet, 2003 ; Simard et al., 2006 ; Barret, 2003) cherchent à formaliser des processus de négociation en amont, des processus de concertation, de médiation, ou de participation, visant à minimiser les risques de conflits.

L'approche juridique s'intéresse principalement, au travers de l'étude des contentieux, à la confrontation des intérêts et à la discussion de l'utilité publique qui s'organisent, notamment dans le cadre de procédures réglementaires, autour des projets d'aménagement (Kirat et Melot, 2006). 
Si la réglementation formalise des procédures d'ajustement des intérêts divergents, ces dernières restent extrêmement ouvertes au jeu des acteurs locaux et à l'état de leur rapport de force. Le rapport de force tourne le plus souvent à l'avantage des intérêts économiques au détriment des intérêts environnementaux (Charbonneau, 2009). À ce niveau, la théorie du bilan coûts-avantages, reposant sur une logique de compensation - notamment des atteintes à l'environnement - constitue le plus souvent un exercice de légitimation du choix d'aménagement soumis à utilité publique (Billet, 2006). Au final, la mise en œuvre de la réglementation aboutit à des situations de « compromis non résolus » (Lascoumes, 1995) en permettant de trouver, à un instant donné, un équilibre entre des logiques d'acteurs divergentes mais sans véritablement résoudre les conflits d'intérêts sous jacents.

La question reste posée pour un aménagement «ordonné » du territoire, de la coordination des différentes logiques territoriales, et de la priorité d'action donnée à telle ou telle initiative. Notre article entend aborder cette question en discutant et en confrontant les réponses de deux grandes règles de coordination : l'une basée sur la hiérarchie des niveaux décisionnels, l'autre -que nous proposons dans ce papier - basée sur la hiérarchie des niveaux d'objectifs.

Une première règle possible de coordination et de priorité d'action est fournie par le principe de hiérarchie des systèmes décideurs. En présence de systèmes décideurs hiérarchiquement dépendants, le problème de coordination peut être résolu par le principe de priorité de l'action de l'unité suprémale (système de niveau supérieur) sur l'action de l'unité infimale (système de niveau inférieur), principe qui traduit la priorité des objectifs et des actions du système sur ceux du sous-système.

$\mathrm{Si}$, en France, il n'existe pas de hiérarchie et de tutelle d'une collectivité territoriale sur une autre (code générale des collectivités territoriales de 1982), une certaine dépendance hiérarchique existe entre niveaux territoriaux (de par l'organisation politico-administrative, le poids réglementaire). Cette relation hiérarchique doit être relativisée : les différents niveaux territoriaux possèdent une liberté décisionnelle et poursuivent des finalités propres. Cette autonomie décisionnelle doit amener à les distinguer des systèmes où la liberté des sous-systèmes n'est que fonctionnelle par rapport au niveau supérieur.

La notion de système hiérarchique multi-échelons (Mesarovic, Macko, Takahara, 1980) est alors utile pour saisir la nature des interrelations entre niveaux décisionnels territoriaux car la dépendance hiérarchique entre systèmes décideurs y est davantage définie comme des contraintes à la réalisation de son propre projet que comme une participation résignée aux finalités du système englobant.

La caractérisation du territoire comme système hiérarchique multi-échelons (section 1) permet ainsi d'insister sur l'autonomie des systèmes décideurs liés aux différents niveaux territoriaux. Cette relative autonomie décisionnelle nécessaire à l'autoréférence est constitutive du système territorial. Au sein d'un système hiérarchique multi-échelons, la performance des sous-systèmes conditionne la performance du système. Dès lors, préserver les conditions d'autoréférence et de fixation de finalités propres au niveau des sous systèmes est nécessaire à la durabilité du système. La caractérisation du territoire comme système hiérarchique multi-échelons ouvre ainsi la voie à une seconde règle possible de coordination et de priorité d'action basée sur la hiérarchie des niveaux d'objectifs poursuivis par les différents systèmes territoriaux : en cas de désaccord sur un projet d'aménagement, la priorité sera donnée au territoire pour lequel le projet présente des enjeux «patrimoniaux » relativement à des enjeux de moindre importance (stratégiques ou de rentabilité) pour l'autre système territoire. 
Il est possible de s'appuyer sur la distinction de la nature identitaire, stratégique et de rentabilité des enjeux liés à un projet d'aménagement (Plottu, 1999) pour donner une formulation opérationnelle d'une règle de coordination basée sur cette hiérarchie des niveaux d'objectifs (section 2). Cette seconde règle de coordination est intéressante notamment dans une optique de développement durable où il peut s'agir de distinguer la nature et l'importance des enjeux liés à telle ou telle option d'aménagement.

Nous sommes donc en présence, potentiellement, de deux règles de coordination, l'une basée sur la hiérarchie des systèmes décideurs, l'autre sur la hiérarchie des niveaux d'objectifs. De la confrontation des deux principes tantôt se renforçant, tantôt s'opposant, il est possible de dégager une règle de décision ou de priorité d'action respectant un objectif d'aménagement durable de l'espace, d'identifier les zones de compensation et de négociation et les zones conflictuelles ( $3^{\mathrm{e}}$ section) entre systèmes décideurs.

\section{2. le territoire comme système hiérarchique multi-échelons}

On peut considérer le territoire comme le résultat, toujours en devenir, des interactions finalisées de l'homme (d'une communauté humaine localisée) avec son milieu. L'aménagement du territoire -le territoire étant fondamentalement défini par la finalité humaine- va dès lors être l'enjeu et le résultat de la confrontation des logiques et finalités de chaque système décideur lié à chacun des systèmes territoires. La référence à la notion de système n'est pas ici fortuite: le territoire peut être défini comme un système et la problématique de l'aménagement du territoire utilement éclairée par une approche systémique, tout territoire apparaissant en effet à la fois comme un tout (composé de territoires) et partie d'un territoire plus vaste, comme tout « système est en même temps un sous-système d'un système, donc un système de système » (Morin, 1977). Il est ainsi permis de parler de système territoire tant les conditions d' «individuation » qui définissent un système (existence d'invariants, présence de frontières, complexification, autonomie par rapport au milieu extérieur, conscience de soi) (Mélèse, 1990) sont au cœur de la notion de territoire (espace où se développe un projet humain).

La référence aux divisions politico-administratives (Europe, État, région, département, intercommunalité, commune) pour établir les différentes échelles territoriales permet d'assimiler chaque système territoire à un système décideur particulier poursuivant des objectifs propres. Cela nous conduit à une représentation hiérarchique du système de gestion territoriale, et l'on pourrait qualifier le territoire de «holon» (Koestler, 1967) territorial, chaque système territoire étant à la fois contenu dans un territoire plus vaste et contenant d'autres territoires comme l'illustre la figure page suivante.

Le concept de système hiérarchique multi-échelons (Mesarovic, Macko, Takahara, 1980) nous semble le plus approprié pour définir l'organisation hiérarchique du système territoire. En effet, le système territoire apparaît comme un système à niveaux multiples, objectifs multiples, et présente ainsi les principales caractéristiques d'un système multi-échelons, à savoir :

(i) le système est composé d'une famille de sous-systèmes en interaction reconnus explicitement,

(ii) certains des sous-systèmes sont définis comme des unités décideurs,

(iii) les unités décideurs sont arrangées hiérarchiquement en ce sens que certaines d'entre elles sont influencées ou commandées par d'autres unités de décision (Mesarovic, Macko, Takahara, p.50). 
Figure 1 : une représentation stratifiée d'un système territoire

État

les régions

les départements

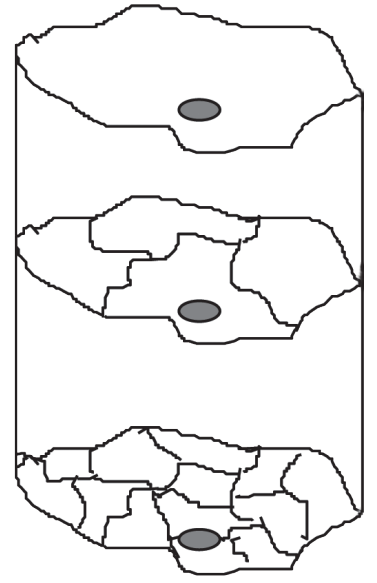

projet d'aménagement du territoire système décideur niveau $\mathrm{n}+1$

systèmes décideur de niveau $\mathrm{n}$

systèmes décideur de niveau n-1

Il faut insister à ce niveau sur une caractéristique fondamentale qui est à la base même de la définition du système hiérarchique multi-échelons, et qui a trait à la dépendance hiérarchique qui existe entre les systèmes décideurs de différentes strates: la relative autonomie des sous-systèmes par rapport au système supérieur. Ainsi, dans un système à niveaux et objectifs multiples, «les unités de niveau supérieur conditionnent mais ne commandent pas complètement les activités de poursuite de leurs objectifs des unités de niveau inférieur » (Mesarovic, Macko, Takahara, 1980, p 51).

Cette relative autonomie -liberté de choix et d'action des sous-systèmes-, n'est pas seulement, dans les organisations humaines, une liberté fonctionnelle mais une liberté décisionnelle entendue ici comme la capacité à déterminer ses propres fins. On retrouve là les notions d'autoréférence, de conscience de soi et d'autodétermination nécessaires à la définition du système territoire. Le territoire est ainsi l'archétype du système hiérarchique multiéchelons. Cette liberté dans la formulation d'objectifs propres précise la nature et relativise l'importance de la dépendance hiérarchique qui lie le sous-système au système suprémal.

Ainsi, la dépendance hiérarchique est davantage synonyme pour le sous-système territoire de contraintes à la réalisation de son propre projet, plus qu'elle n'indique la participation résignée du sous-système au projet de l'unité de décision suprémale (du système territoire d'ordre supérieur). Nous considérons ainsi au minimum que le système suprémal fixe, du moins en partie, les règles du jeu pour l'action des sous-systèmes et trace les limites du possible pour les choix des unités de décision infimales. La logique de la régulation/équilibration (Thireau, 1993) est ainsi parfaitement adaptée à l'appréhension des relations entre systèmes territoriaux. La structuration de l'espace est ainsi inévitablement l'occasion de confrontation des logiques de ces systèmes décideurs qui projettent sur l'espace l'image désirée de leur territoire.

Dès lors, la problématique de l'aménagement pose fondamentalement la question des interrelations entre ces systèmes décideurs, de la compatibilité et de la coordination des objectifs et des projets sur l'espace portés par chaque système décideur dans la poursuite de ses finalités propres. Le territoire étant défini comme système multi-échelons, on peut, 
conformément à la théorie des systèmes, énoncer deux principes généraux qui vont guider et encadrer les modalités de relations entre systèmes territoires.

\subsection{Le droit d'intervention ou le principe de priorité de l'action de l'unité suprémale} (système de niveau supérieur)

"Les opérations d'un sous-système de n'importe quel niveau sont directement et explicitement influencées à partir des niveaux les plus élevés (...) Cette influence est obligatoire sur les niveaux les plus bas et traduit une priorité d'importance, dans les actions et objectifs des niveaux élevés. » (Mesarovic, Macko, Takahara, 1980, p37).

Il peut y avoir deux sortes d'intervention du système décideur de niveau supérieur: une intervention pré-décisionnelle: l'unité suprémale essaie de coordonner les actions des unités infimales; et une intervention post-décisionnelle: l'unité suprémale essaie de corriger l'action de l'unité infimale ou au contraire de récompenser celle-ci. On retrouve ici la logique de la régulation à laquelle nous faisions précédemment référence. L'information est une variable clef de contrôle du système sur les sous-systèmes; la relation hiérarchique entre unités décideurs peut à ce niveau s'analyser comme une relation de principal à agent.

Le premier principe que nous nommerons « principe hiérarchique » fournit une réponse à notre interrogation sur les modalités de coordination des actions et des objectifs conflictuels des systèmes décideurs, en donnant la priorité aux objectifs poursuivis par le système territoire englobant sur ceux des sous-systèmes. Le système politico administratif français (marqué par un fort degré de centralisation et de commandement «par le haut») et les objectifs assignés à l'aménagement du territoire (redistribution des richesses) jusqu'au début des années 80 favorisent, en cas de conflit entre systèmes décideurs, la priorité des objectifs du système sur ceux du sous-système. De fait, le principe hiérarchique a ainsi régulé les relations entre les différents niveaux territoriaux et déterminé le processus décisionnel concernant les actions sur le territoire. Le principe hiérarchique s'imposait naturellement comme mode de coordination efficace des actions des systèmes décideurs sur le territoire au vue de l'organisation politicoadministrative, des objectifs de l'aménagement et de la croyance en un développement «par le haut », qui prévalaient en France jusqu'au début de la décennie 80.

\subsection{L'interdépendance des performances}

«Bien que la priorité de l'action soit orientée en descendant à la façon d'un commandement, le succès du système global, et, en vérité, celui des unités de tous les niveaux, dépend des performances de toutes les unités du système. » (Mesarovic, Macko, Takahara, 1980, p 37).

Il existe en particulier une dépendance des performances du niveau le plus élevé des performances réelles des niveaux les plus bas. Le succès de l'unité suprémale dépendra ainsi des actions et des performances des unités infimales. La conséquence immédiate est que le système ne peut durablement se désintéresser de la performance et des intérêts des unités infimales. Le système ne peut en particulier aller à l'encontre des intérêts vitaux des soussystèmes sans courir le risque de péricliter. Le système doit veiller à préserver les conditions d'autoréférence et la liberté décisionnelle des sous-systèmes. Cette caractéristique ouvre sur un second principe de priorité d'action basée sur la considération de la nature des enjeux sous tendant la position des territoires vis-à-vis du projet d'aménagement. 


\section{Principe hiérarchique des niveaux d'objectifs basés sur l'identification des enjeux identitaires, stratégiques, de rentabilité du projet d'aménagement}

Jusqu'où la poursuite des objectifs de l'unité suprémale peut-elle s'opposer aux objectifs poursuivis par les unités infimales ? La poursuite d'objectifs peu importants par le système englobant doit-elle s'imposer (d'une façon systématique ?) à la poursuite d'objectifs plus fondamentaux d'un système inférieur ? L'interdépendance des niveaux de performance pose les limites d'application du principe de priorité d'action à donner au système sur le sous- système.

Une difficulté est de pouvoir qualifier (selon quels critères ?) un objectif « de peu important », «d'important » ou de «fondamental ». Afin de qualifier la nature des objectifs que poursuit chaque système décideur et qui vont motiver le projet d'équipement ou d'aménagement du territoire, il est possible de se référer aux trois principaux types d'objectifs poursuivis par une organisation humaine (Mélèse, 1990 ; Atlan, 1979) :

- un objectif de reproduction ;

- un objectif de développement ;

- un objectif d'optimisation du temps présent.

À ces objectifs, on peut associer trois problématiques de choix auxquelles toute organisation humaine, et ici tout système décideur lié à un territoire, peuvent être confrontés:

- des choix identitaires. Ces choix portent sur les éléments clefs qui font l'identité d'un territoire et qui contribuent au sentiment d'appartenance d'une population à ce territoire. Ces éléments clefs sont indispensables à l'objectif de pérennité et de re-production du système territoire. Ce type de choix sera qualifié d'ordre III.

- des choix stratégiques. On parlera de choix stratégiques lorsque des éléments déterminant des potentialités de développement du territoire sont en jeu. Les choix d'aménagement et de valorisation des ressources locales constituent de bons exemples de choix stratégiques. (choix qualifiés d'ordre II).

- des choix de rentabilité qui relèvent d'une recherche d'optimisation du temps présent, recherche de la rentabilité maximale à très court terme (principe de moindre effort). Ces choix portent sur des éléments qui n'obèrent pas les possibilités de développement du territoire. Choix qualifiés de type I.

En différenciant les choix identitaires, stratégiques et de rentabilité, on souligne la distinction fondamentale entre les problématiques de choix à l'intérieur d'un espace de choix et la problématique de choix d'un espace de choix; distinction mise en évidence notamment par O.Favereau (1982) et fondamentale pour l'appréhension des problématiques de l'environnement (Plottu, 1999). On peut également retrouver cette distinction dans les travaux fondamentaux de Ansoff (1965) sur le management stratégique et en particulier dans la distinction entre l'efficience stationnaire et l'adaptabilité stratégique ou structurelle.

Ainsi, il existe en particulier une dépendance hiérarchique entre les différents types de choix: les choix identitaires (d'ordre III) doivent normalement déterminer l'espace des possibles pour les choix d'ordre II (le choix d'une stratégie de développement d'un territoire ne peut se concevoir que si au préalable un choix identitaire (quelle est l'image désirée du territoire ?) a été défini. De même, le choix d'ordre II déterminera à son tour le champ des possibles pour les choix d'ordre I (la recherche de maximum de rentabilité doit en effet être compatible avec le choix stratégique). Le choix de type I est ainsi hiérarchiquement 
dépendant du choix de niveau supérieur (de type II), la problématique de choix de type II apparaissant elle-même hiérarchiquement dépendante du niveau de choix d'ordre supérieur (de type III). En cas d'objectifs concurrents, on peut ainsi énoncer un principe de "priorité de la problématique de choix d'ordre supérieur »: les objectifs d'ordre III sont prioritaires sur les objectifs d'ordre II, eux-mêmes étant prioritaires sur les objectifs d'ordre I.

Tous les enjeux liés à une décision -décision qui concerne ici un projet d'aménagement du territoire- peuvent être décomposés en enjeux identitaires, stratégiques ou de rentabilité. Tout projet s'exerçant sur l'espace va ainsi signifier pour le système décideur des enjeux identitaires, stratégiques ou de rentabilité, en entraînant la création/transformation ou destruction de relations constitutives du territoire d'ordre I,II ou III.

Les relations finalisées entre l'homme et le milieu, relations qui définissent un territoire, peuvent en effet être décomposées en relations identitaires, stratégiques ou de rentabilité selon qu'elles déterminent l'identité (la structure) du territoire (relations de type III), qu'elles constituent un potentiel de développement pour le territoire (relations de type II) ou qu'elles ne représentent que des enjeux de rentabilité immédiate (relations de type I). Chaque système territoire peut ainsi être décrit en termes de relations d'ordre I,II,III.

Figure 2 : le système territoire: des relations identitaires, stratégiques et de rentabilité

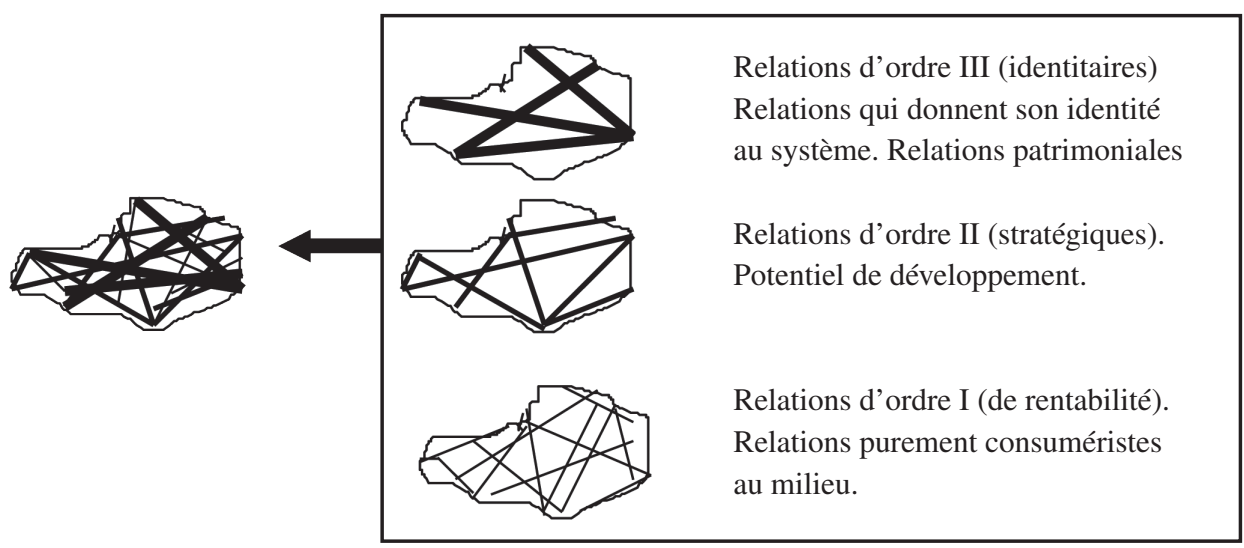

Toutes les relations n'ont pas la même importance dans la structuration et l'individuation du territoire. Ainsi, certaines relations (que nous qualifierons alors d'identitaires -d'ordre III-) sont indispensables à l'autoréférence et conscience de soi du territoire, c'està-dire qu'elles apparaissent fondamentales dans la structuration et l'individuation du territoire, dans l'émergence de finalités propres qui sont à la base de la définition même d'un territoire. Les relations d'ordre I (de rentabilité) ne participent pas directement, à l'opposé, à l'identité du territoire. Ces relations purement consuméristes qu'entretient l'homme avec son milieu ne sont pas indispensables à la définition du système territoire. Les relations d'ordre II, quant à elles, assurent la reproduction du système territoire dans le temps, elles constituent des potentiels de développement pour le territoire. Les relations d'ordre III apparaissent ainsi plus importantes que les relations d'ordre II pour le système territoire, les relations d'ordre II étant elles-mêmes plus importantes que les relations d'ordre I. 
Tout projet d'aménagement, et plus généralement toute intervention sur le territoire, vont ainsi mettre en jeu des relations d'ordre I, II, III. Le projet d'aménagement va donc signifier, pour chaque système territoire, des enjeux identitaires, stratégiques ou de rentabilité. L'évaluation des effets du projet sur le territoire doit aussi tenir compte de la nature des relations mises en cause.

En effet, il ne sera évidemment pas équivalent de créer/transformer/détruire une relation d'ordre III et une relation d'ordre I. Il n'existe pas par exemple de compensation possible entre la perte d'une relation structurante du territoire (d'ordre III) et la création d'une relation d'ordre I. Le problème, qui apparait ici, est dans l'identification des relations mises en cause par le projet d'aménagement et ceci au niveau de chaque système territoire affecté par le projet. En effet, ce qui apparaîtra comme création/destruction d'une relation de type I au niveau d'un système territoire pourra être perçu du point de vue d'un autre système territoire comme une création/destruction d'une relation de type III.

Prenons l'exemple d'un projet autoroutier impliquant la destruction d'une forêt. Cet exemple est inspiré d'un cas réel, celui du choix du tracé autoroutier de l'A85 (ToursVierzon) sur le territoire de la commune de Pruniers-en-Sologne (Plottu, 1999; Plottu et Plottu 2007) : les services techniques de l'État et les acteurs locaux sont en conflit quant au choix du tracé autoroutier. Si les parties en présence s'accordent sur la réalité et la mesure de l'impact physique du tracé (la perte de forêt), ils ont une appréciation divergente de la nature des impacts et des enjeux soulevés par le tracé sur le territoire. Pour le système territoire englobant (les services techniques de l'État), la construction de l'autoroute constitue une relation de type II (un potentiel de développement pour le territoire), la destruction de la forêt n'étant perçue que comme une destruction de type I (la perte liée à la forêt est strictement financière -production de bois- cette forêt n'étant qu'une forêt parmi les innombrables forêts du territoire). Or, pour le sous système territorial (le maire et la population de la commune concernée), cette forêt constitue un élément structurant: située au centre du territoire communal, la forêt doit faire l'objet d'un projet de valorisation par la création d'un conservatoire des animaux de la ferme. Ce projet vise à « rapprocher » les différentes zones d'habitation de la commune qui sont éparpillées sur le territoire, et à préserver ainsi l'unité et l'identité rurale de la commune. Cette forêt constitue donc un élément patrimonial. (de type III) du point de vue de la commune. Par contre, l'autoroute n'aura pas de retombées économiques particulières pour le territoire au niveau local; tout au plus peut-on espérer quelque avantage financier (relation de type I). Ce qui est ainsi création d'une relation d'ordre II et destruction d'une relation d'ordre I à un niveau territorial est, à un autre échelon territorial, création d'une relation d'ordre I et destruction d'une relation d'ordre III, ce qui pose évidemment problème au niveau de l'évaluation des impacts du projet d'aménagement. La destruction d'une forêt ne fait pas résonner les mêmes enjeux et raisonner la même évaluation selon l'échelon territorial considéré. La qualification de la nature des impacts du projet sur le territoire par différenciation des choix identitaires, stratégiques et de rentabilité a permis d'objectiver les divergences qui opposaient services techniques de l'État et acteurs locaux. Le croisement des intérêts contradictoires selon la nature de l'impact du projet donne à voir les marges de négociation possibles entre les différents protagonistes.

La problématique de l'aménagement pose ainsi un double problème : premièrement, celui de l'identification de la nature des enjeux de l'aménagement pour chaque système décideur du système territorial multi-échelons; deuxièmement celui de la confrontation et de la coordi- 
nation des logiques territoriales et projets développés par les différents systèmes décideurs. À ce niveau, le principe hiérarchique suggéré par la théorie des systèmes de priorité du système décideur suprémal nous a fourni un premier mode de coordination des interrelations conflictuelles entre systèmes décideurs: la priorité des objectifs et actions du système sur ceux des sous-systèmes. La hiérarchie des types d'objectifs d'ordre III, II, I nous indique un second mode de coordination possible des objectifs conflictuels des systèmes décideurs : la priorité des objectifs (et enjeux) identitaires sur les objectifs stratégiques et de rentabilité.

Cette priorité hiérarchique des objectifs d'ordre III sur les objectifs d'ordre II et I permet ainsi d'éclairer théoriquement la question de la coordination des actions et objectifs conflictuels des systèmes décideurs. Considérons ainsi un système décideur $\mathrm{A}$ (lié à un système territoire) favorable à un projet d'équipement/d'aménagement du territoire. Les enjeux du projet d'aménagement pour le système décideur A seront d'ordre identitaire, stratégique ou de rentabilité. Soit un système décideur B opposé au projet d'aménagement précédent: les impacts (négatifs pour B) du projet seront de même d'ordre identitaire, stratégique ou de rentabilité. En l'absence de dépendance hiérarchique entre les deux systèmes décideurs, c'est-à-dire que les deux sous-systèmes territoires appartiennent à un même échelon territorial (une même strate), le principe hiérarchique de priorité du système suprémal ne peut s'appliquer. Le principe de priorité du niveau d'objectif le plus élevé donne alors la priorité d'action au système décideur pour lequel les enjeux liés au projet sont d'ordre le plus élevé (relativement aux enjeux pour l'autre système territoire). Le tableau suivant indique à quel système décideur (A ou B) est donnée la priorité d'action selon le principe de priorité du niveau d'objectif le plus élevé:

Tableau 1 : Priorité d'action selon la hiérarchie des niveaux d'objectifs

Nature
de l'impact

Territoire A

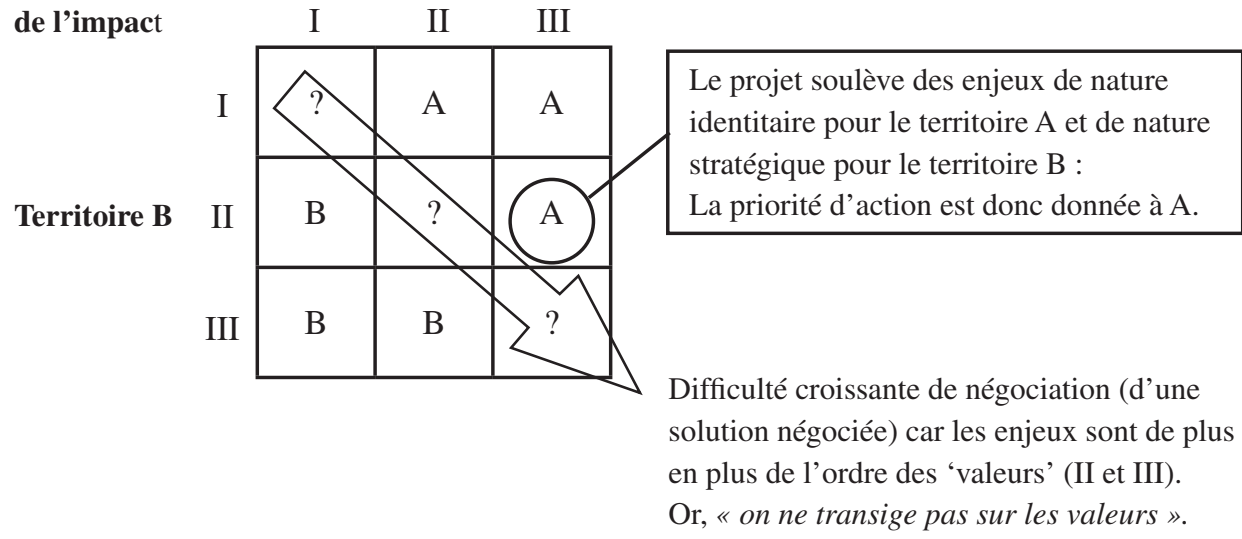

Le principe de priorité hiérarchique des niveaux d'objectifs ne donne pas d'indication lorsque les enjeux pour chaque système décideur sont de même ordre (I/I; II/II et III/ III). Notons simplement la difficulté croissante de négociation (élaboration d'une solution négociée) lorsque les enjeux liés à l'aménagement sont d'ordre élevé (II ou III) pour les deux systèmes décideurs. En effet, s'il est possible de s'entendre et de négocier sur des enjeux d'ordre I, il devient impossible de transiger sur les objectifs identitaires. 
Si nous envisageons le même problème mais en supposant maintenant une dépendance hiérarchique entre les systèmes décideurs: imaginons par exemple que le système territoire $B$ soit un sous-système du système territoire $A$. (A est le système décideur suprémal, B le système décideur infimal selon la terminologie de Mesarovic, Macko, Takahara (1980). Si nous appliquions le principe hiérarchique donnant la priorité d'action au système décideur suprémal, le résultat de la confrontation des objectifs conflictuels serait immédiat: les objectifs poursuivis par A (et donc le projet porté par A) s'imposent à ceux de B; ceci quelle que soit la nature des effets du projet pour chaque système décideur.

Tableau 2 : Priorité d'action selon la hiérarchie des systèmes décideurs

\begin{tabular}{|c|c|c|c|c|}
\hline & & A sy & e de & au $\mathrm{n}$ \\
\hline & & I & II & III \\
\hline & I & A & A & A \\
\hline $\begin{array}{c}\text { B } \\
\text { système }\end{array}$ & II & A & A & A \\
\hline & III & A & A & A \\
\hline
\end{tabular}

L'intérêt de distinguer dans un tel problème de décision les enjeux d'ordre I, II, III pour chaque système territoire apparât ici maintenant plus clairement: ainsi, si l'application du principe de priorité d'action de l'unité suprémale A sur l'unité de décision infimale $\mathrm{B}$ demanderait que l'on obtienne le tableau précédent (en conflit, A emporte la décision), il peut apparaître choquant qu'une décision du système suprémal ne relevant que d'une recherche de rentabilité doive systématiquement s'imposer, ceci même au prix de la disparition de relations identitaires ou patrimoniales au niveau du sous-système, en d'autres termes de relations structurantes du soussystème territoire.

Il semble bien ainsi nécessaire de tenir compte du type d'objectifs poursuivis, via le projet sur le territoire, par les différents systèmes décideurs et d'évaluer la nature des enjeux de l'aménagement à chaque niveau territorial. Nous n'avons plus dès lors une seule hiérarchie celle des systèmes décideurs- pour donner la priorité aux objectifs et à l'action d'un système territoire, il faut aussi considérer la hiérarchie des objectifs poursuivis.

\section{Confrontation des deux règles de priorité d'action issues de la hiérarchie des systèmes décideurs et des niveaux d'objectifs}

Si l'on considère maintenant simultanément les deux principes et si l'on couple ainsi le principe de priorité des objectifs du système suprémal et le principe de priorité $\mathrm{du}$ niveau d'objectif le plus élevé, on obtient alors le tableau ci-dessous des priorités d'action et d'objectifs: 
Tableau 3 : Priorité d'action issue de la confrontation des deux principes hiérarchiques

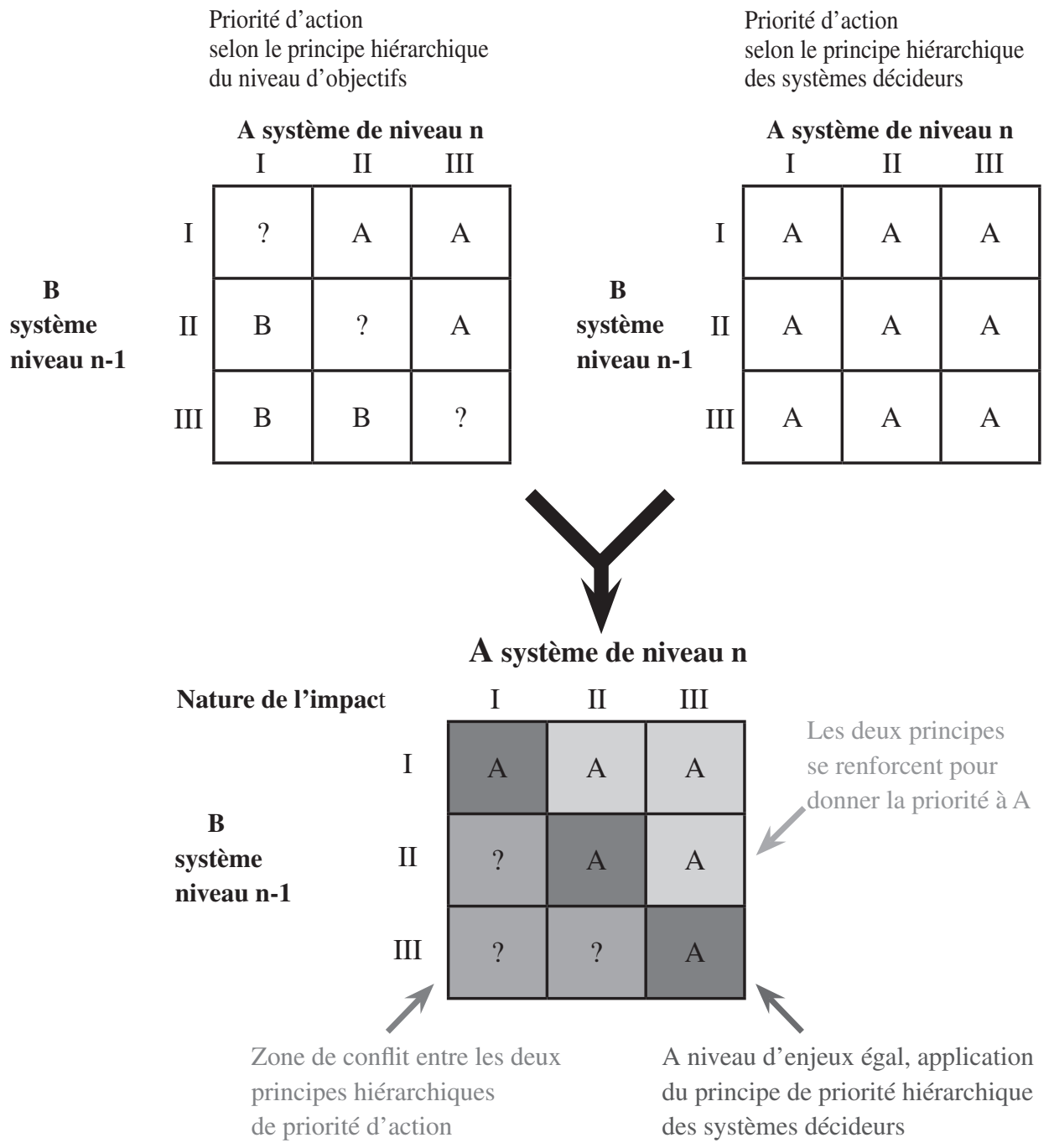

La première ligne du tableau ne présente pas de difficulté particulière, les deux ordres de priorité se renforçant: le système décideur suprémal poursuit des objectifs d'ordre supérieur à ceux poursuivis par le sous-système (objectifs de rentabilité). La priorité d'action est donc donnée au système $\mathrm{A}$. Les enjeux négatifs pour le système $\mathrm{B}$ étant d'ordre $\mathrm{I}$, on peut escompter une faible opposition de B à la mise en œuvre du projet. Par contre, la priorité d'action donnée à A dans les autres cas soulève inévitablement de forts risques d'opposition de $\mathrm{B}$ à la mise en oeuvre des projets de $\mathrm{A}$. En effet, le système territoire $\mathrm{B}$ ne peut accepter de supporter les effets négatifs de la mise en oeuvre du projet, ces effets négatifs intéressant des relations stratégiques (de type II) ou identitaires (de type III) de son système territoire. 
Enfin, une zone d'indétermination de la priorité à accorder à l'un ou l'autre système décideur apparaît lorsque les deux ordres de priorité préconisés par la hiérarchie des systèmes décideurs et la hiérarchie des types d'objectifs s'opposent, c'est-à-dire lorsque le système décideur suprémal poursuit des objectifs I ou II qui s'opposent aux objectifs d'ordre supérieur (II ou III) poursuivis par le sous-système décideur.

Les objectifs d'ordre I de l'unité suprémale doivent-ils s'imposer alors que les effets négatifs pour le sous-système sont d'ordre II voire III ? Il n'existe pas de réponse définitive à cette question. Donnera t-on la priorité au principe hiérarchique des systèmes décideurs ou au contraire à la hiérarchie des niveaux d'objectifs ?

Dans ce dernier cas, la priorité de considérations identitaires ou stratégiques d'un système décideur inférieur s'imposerait ainsi aux objectifs inférieurs (de rentabilité) poursuivis par un système décideur supérieur. Le système décideur inférieur se trouverait doté d'un droit d'intervention prioritaire relativement au système décideur supérieur. Le principe de subsidiarité qui entend donner la priorité à l'initiative «par le bas » et à une procédure d'appréciation «montante » des décisions, trouverait alors ici un champ d'application privilégié. En distinguant ce qui relève d'objectifs ou d'enjeux identitaires, stratégiques et de rentabilité, le principe de priorité hiérarchique des niveaux d'objectifs et le principe de subsidiarité pourraient alors mutuellement se doter d'une légitimité et d'une opérationalité face à d'autres modes de partage des compétences et de coordination des objectifs des systèmes décideurs, modes qui relèvent davantage d'une logique de l'intégration.

L'objectif de durabilité nécessite a minima de s'assurer des conditions d'existence du système. Dans une optique d'aménagement durable du territoire, la règle de priorité des niveaux d'objectifs s'impose à la règle basée sur la hiérarchie des niveaux décideurs. La priorité d'action du système sur le sous système s'applique alors lorsque les enjeux soulevés par le projet d'aménagement sont de même niveau. Cette priorité lexicographique donnée à la hiérarchie des niveaux d'objectifs permet de lever les zones d'indétermination issue de l'opposition des deux principes hiérarchiques (les «? » du tableau 3). Le tableau 4 résume ainsi, en cas de conflits autour de l'opportunité d'un projet d'aménagement, les priorités d'action à donner à tel ou tel territoire dans une optique d'aménagement durable du territoire.

Tableau 4 : Proposition de règle de priorité d'action dans une optique d'aménagement durable du territoire

\section{A système de niveau n}

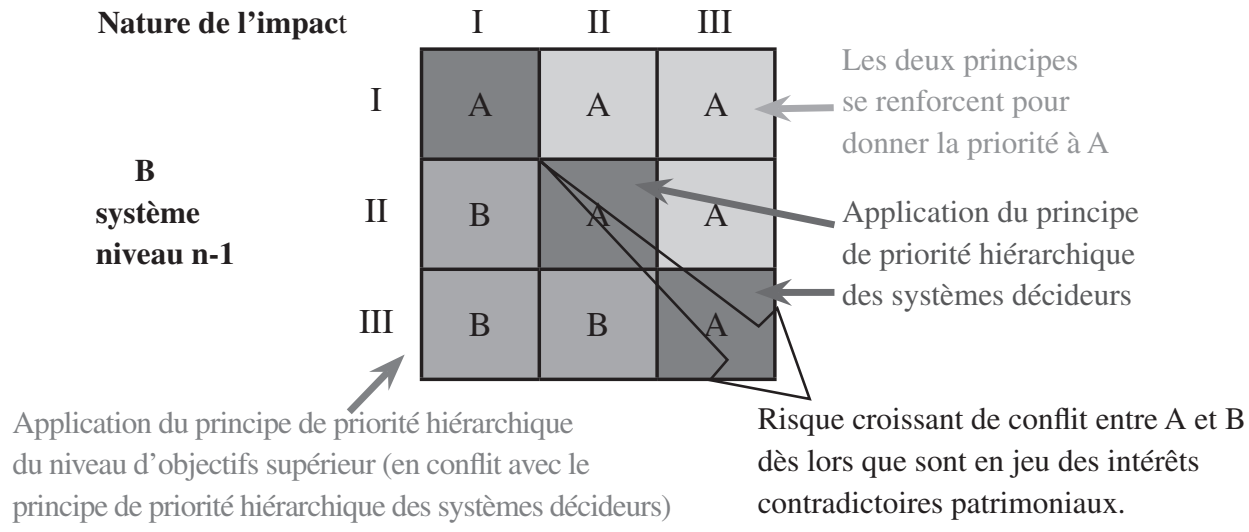


Les risques de conflits seront d'autant plus forts que sont en jeu pour chacun des deux systèmes décideurs des enjeux d'ordre supérieur (stratégiques et, a fortiori, patrimoniaux). A contrario, les probabilités d'issue négociée en l'absence de règles de priorité d'action coercitives seront d'autant plus élevées si les enjeux pour au moins l'un des deux systèmes décideurs sont d'ordre I. Quatre situations peuvent être identifiées (la compensation, la négociation, le conflit, le blocage) repérées dans le tableau et le schéma suivants :

Tableau 5 : Carte des blocages / conflits / négociation /compensations (cartographie BCNC)

Conflits d'objectifs des systèmes décideurs A et B

\begin{tabular}{|c|c|c|c|c|}
\hline \multirow[b]{2}{*}{$\begin{array}{l}\text { Système } \\
\text { décideur B }\end{array}$} & & $\begin{array}{l}\text { Nature de l'in } \\
\text { Système } \\
\text { décideur A } \\
\text { I } \quad \text { II }\end{array}$ & act & \\
\hline & I & Négociation & Négociation & Compensation \\
\hline & II & Négociation & Conflits & Conflits \\
\hline & III & Compensation & Conflits & Blocage \\
\hline
\end{tabular}

Schéma 3 : zonage des blocages / conflits / négociation /compensations pour un projet autoroutier

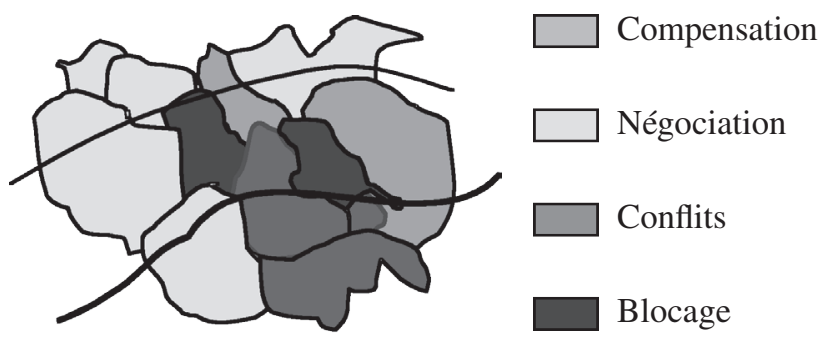

Le tableau ci-dessus permet de visualiser l'ensemble des combinaisons possibles d'enjeux pour deux systèmes décideurs en présence. Plus les enjeux apparaissent importants pour les deux parties, plus les risques de conflits sont forts : dans le cas où les d'enjeux sont d'ordre III / III pour les deux parties, les risques de blocages sont maximums. Aucun des deux systèmes territoires ne peut renoncer et les enjeux « patrimoniaux » relèvent du non négociable. A contrario, plus les enjeux apparaissent relever de considérations de recherche de rentabilité, plus les acteurs pourront négocier et transiger autour de la faisabilité ou non du projet (cas I / I, I/II ou II/I). Lorsque les enjeux sont d'ordre stratégique et/ou patrimonial (cas II/II ou II/III), les risques de conflit sont nombreux. Dans le cas I/III, la probabilité de compensation est forte. Le porteur d'enjeux I acceptera une compensation et le système décideur poursuivant des objectifs de niveau III sera enclin à accepter de compenser pour faire passer son projet. 
Il serait possible, en ayant recours aux Système d'Information Géographique (SIG) notamment, et sur la base de l'identification préalable de la nature des enjeux d'un projet particulier sur un territoire, d'établir ex ante un schéma des zones de blocages /conflits/ négociation/compensation entre deux systèmes décideurs en présence. Le schéma 3 est construit à partir de l'identification des enjeux pour les territoires de deux tracés (par exemple, tracés autoroutiers, tracés de ligne à grande vitesse, ...). Il permet de visualiser et d'anticiper les risques d'opposition locale à l'un ou l'autre tracé.

L'identification pour chaque groupe d'acteurs des enjeux identitaires, stratégiques, de rentabilité soulevés par un projet permet ainsi de mieux comprendre et d'anticiper un certain nombre de conflits qui se forment autour des projets d'aménagement. La qualification et la discussion collective du type d'enjeu peuvent permettre une meilleure compréhension des positions de chacun et la mise en évidence des marges et des limites de négociation.

\section{Conclusion}

«C'est de la superposition des compétences et des territoires que naît le risque de chevauchement des actions et de confusion des légitimités, tandis que se multiplient les soucis existentiels, chaque échelon revendiquant le droit à s'occuper de tout et ne trouvant de limites que dans la contrainte budgétaire. L'accent mis par de plus en plus de collectivités sur le développement durable des territoires qui leur permet d'investir quasiment tous les champs de l'action publique en constitue une illustration évidente» (Piron, 2006, p.307).

L'équilibre territorial des pouvoirs et la cohérence des politiques conduites par les différents niveaux territoriaux posent la question des règles sur lesquelles organiser la coordination et de la gestion des conflits des projets de territoire.

La rationalisation/diminution des échelons de gestion du territoire a ses limites et l'affirmation du rôle de coordination des préfets ou la mise en œuvre de la notion de collectivité « chef de file » (révision constitutionnelle du 28 mars 2003, Art.72, alinéa 5) dans la coordination des politiques soulève des difficultés dans un pays où la hiérarchie entre collectivités territoriales n'existe pas.

Mais les choses évoluent. Le rapport Fritsch (2008) insiste sur la nécessaire hiérarchisation des niveaux entre les collectivités pour une meilleure efficacité des interventions publiques sur le territoire. La hiérarchie des systèmes décideurs constitue alors un mode tout désigné de coordination des logiques territoriales. Ce qui n'est que donner la priorité $\mathrm{du}$ global sur le local et affirmer la primauté de l'intérêt général sur l'intérêt particulier.

Mais de quels types d'intérêt s'agit-il ? La règle de coordination basée sur la hiérarchie des niveaux d'objectifs que nous proposons repose précisément sur l'identification et la comparaison de la nature des enjeux des systèmes décideurs. On ne peut traiter indifféremment des enjeux patrimoniaux touchant à l'identité même d'un territoire et des enjeux de recherche de rentabilité de court terme.

Finalement, le point de vue du système englobant poursuivant des objectifs de rentabilité de court terme doit-il être prioritaire par rapport au point de vue du sous-système défendant des intérêts patrimoniaux ? Dans une optique d'aménagement durable du territoire, la réponse que nous apportons donne la priorité à la hiérarchie des niveaux d'objectifs. Ce faisant, elle soulève la question des processus d'évaluation et de décision basés sur l'expression et la discussion des enjeux entre différents échelons territoriaux. Cela 
suppose une place plus importante laissée à des formes d'évaluation participative, ce qui ne répondrait finalement qu'aux recommandations et aux cadres de référence nationaux (Bardou, Duport, Senna, 2005) cherchant à accompagner les démarches et les projets territoriaux de développement durable.

\section{Références}

Ansoff H.I., 1965, Corporate Strategy, Mc Graw Hill, New York.

Atlan, H., 1979, Entre le cristal et la fumée. Essai sur l'organisation du vivant, Paris, Seuil

Bardou M., Duport L., Senna P., 2005, Projets territoriaux de développement durable et agendas 21 locaux : un cadre pour l'action, Paris, Ministère de l'Écologie, de l'Énergie, du Développement Durable et l'Aménagement du Territoire (MEEDDAT), octobre.

Barret P. (Geyser), 2003, Guide pratique du dialogue territorial, Concertation et médiation pour l'environnement et le développement durable local, Fondation de France.

Billé R., Mermet L. (ss la dir.), 2003, Concertation, Décision et environnement. Regards croisés, Volume II, Paris, La Documentation Française.

Billet P., 2006, « La prise en compte de la faune sauvage dans le cadre des activités et procédures d'aménagement, de gestion et d'utilisation des sols », Actes du colloque «Gestions durables des espèces animales (mammifères, oiseaux) - Approches biologiques, juridiques et sociologiques» (Paris 15-17 novembre 2004), in Natures- Sciences-Sociétés 2006, vol. n²14, suppl. pp. S. 13 à S. 21.

Charbonneau S., 2009, « Les projets autoroutiers en cours maintenus malgré le Grenelle », Les annales de la voirie, $\mathrm{n}^{\circ} 135$, mai-juin, pp.14-16.

Favereau O., 1982, Risques, irréversibilités et fondements de l'analyse coût-avantage, Thèse pour le doctorat de 3ème cycle, université Paris X Nanterre.

Fritsch B., 2008, «Investissement public local et maîtrise de la dépense publique. De la maîtrise de la dépense à la révision des modes de conduite de l'action publique territoriale », Les cahiers de l'Institut de la Décentralisation, $\mathrm{n}^{\circ} 10$, octobre.

Kirat T. et Melot R., 2006, « Du réalisme dans l'analyse économique des conflits d'usage : les enseignements de l'étude du contentieux dans trois départements français (Isère, Loire-Atlantique, Seine-Maritime). », Développement durable et territoires [En ligne], Dossier 7 : Proximité et environnement, mis en ligne le 10 mai 2006, Consulté le 07 septembre 2009. URL : http://developpementdurable.revues.org/index2574.html

Koestler A., 1967, The Ghost in the Machine, Hutchinson.

Lascoumes P., 1995, « Les arbitrages publics des intérêts légitimes en matière d'environnement. L'exemple des lois Montagne et Littoral », Revue française de science politique, vol.45, n 5 , pp.396-419.

Melé P., Larrue C., Rosenberg M. , 2003, Conflits et territoires, Presses universitaires François-Rabelais, Maison des sciences de l'homme « Villes et territoires ».

Mélèse J., 1990, Approches systémiques des organisations, Paris, Les Éditions d'Organisation.

Mesarovic M.D., Macko D., Takahara Y., 1980, Théorie des systèmes hiérarchiques à niveaux multiples, Paris, Économica.

Morin E., 1977, La méthode, la nature de la nature, Tome I, Paris, Seuil.

Piron M., 2006, « Gouverner en France : Quel équilibre territorial des pouvoirs ? », Rapport d'information au nom de la commission des Lois sur l'équilibre territorial des pouvoirs, Assemblée Nationale, XIIe législature, $\mathrm{n}^{\circ} 2881,22$ février, $354 \mathrm{p}$.

Plottu E., 1999 Environnement : Principe et méthodologie de l'Evaluation Hiérarchique. Un cadre pour le développement durable et l'analyse de projets, Thèse pour le doctorat en Sciences Economique, Université de Rennes 1. 
Plottu E., Plottu B., 2007, « The concept of Total Economic Value of environment : A reconsideration within a hierarchical rationality», Ecological Economics, 61, pp.52-61.

Simard L. , Lepage L. , Fourniau J-M., Gariépy M., Gauthier M. (ss la dir.), 2006, Le débat public en apprentissage. Aménagement et environnement. Regards croisés sur les expériences française et québécoise, Ed. L'Harmattan.

Thireau V., 1993, «Vers un renouvellement du rôle de l'espace dans la dynamique des territoires », Revue d'Economie Régionale et Urbaine, ${ }^{\circ} 2$, pp 261-287. 
